\title{
El desarrollo comunitario de nuevo coronillas. municipio de Charo, Michoacán. Enfoque dual
}

\author{
Celso Rivera Hernández \\ criverah2008@gmail.com \\ Docente-Promotor de la Brigada de Educación \\ para el Desarrollo Rural No 104 \\ Charo, Michoacán - México.
}

\section{RESUMEN}

El desarrollo comunitario dual, es una propuesta emergente de la investigación social, que mediante la intervención educativa contribuye decididamente en la solución de problemas que aquejan a la población rural; el efecto dual se logra cuando los actores comunitarios, sincrónicamente y por sí mismos, logran satisfacer sus necesidades apremiantes y potencian su disponibilidad a la resiliencia. Esta propuesta surge de la reflexión que después de 30 años de creado el primer programa, de gran cobertura, para la asistencia económica en beneficio de los sectores más desfavorecidos, y que luego de 85 años de fundada la primera institución para financiamiento campesino, los casos de éxito son escasos; a esas acciones las desfavorecen la asignación de beneficios aislada de todo ejercicio educativo. Recapitulando en nuestra propia praxis, rememoramos que los efectos de la intervención educativa dual, se manifiestan en evidencias de transformación anímica y económica, tanto en las personas como en su colectividad. Desde Rousseau hasta Freire y Rafael Ramírez, se da cuenta de la importancia de la educación en el devenir social.

Palabras clave: comunitario; desarrollo; dual; educación; resiliencia. 


\title{
The community development of nuevo coronillas. municipality of Charo, Michoacán. Dual approach.
}

\begin{abstract}
Dual community development is an emerging proposal of social research, which through educational intervention contributes decisively to the solution of problems that afflict the rural population; the dual effect is achieved when community actors, synchronously and on their own, manage to meet their pressing needs and enhance their availability for resilience. This proposal arises from the reflection that after 30 years of creating the first program, with great coverage, with great coverage, for economic assistance for the benefit of the most disadvantaged sectors, and that after 85 years of founding the first institution for rural financing, the cases of success are rare; These actions are disadvantaged by the allocation of benefits isolated from any educative . Recapitulating in our own praxis, we recall that the effects of dual educational intervention are manifested in evidence of animosity and economic transformation, both in people and in their community. From Rousseau to Freire and Rafael Ramírez, he realizes the importance of education in social development.
\end{abstract}

Keywords: community; development; dual; resilience; intervention.

Artículo recibido: 10 Setiembre. 2021 Aceptado para publicación: 15 Octubre. 2021 Correspondencia: criverah2008@ gmail.com Conflictos de Interés: Ninguna que declarar 


\section{INTRODUCCIÓN}

Las políticas sociales, en México, por lo general se caracterizan por ser de asistencia, carentes del componente educativo, generador y reanimador de conciencia ciudadana. A excepción de escasas acciones, por mucho insuficientes, mediante las cuales se equipa con maquinaria o equipo a los participantes en cursos de capacitación para el trabajo, administración y comercialización, los esfuerzos por abatir la pobreza son nulos. Pues aunque es cierto que los beneficiados por la asistencia, temporalmente mejoran sus carencias, también es muy cierto que en ellos se forma un sentimiento de dependencia.

Precisamente, este planteamiento de desarrollo comunitario con enfoque dual, trasciende los criterios obsoletos conque tradicionalmente se atienden las necesidades comunitarias e implica el concurso de disciplinas soslayadas por el tecnicismo utilitarista. Revisa las propuestas de instituciones, organizaciones y especialistas del tema; identifica rasgos de los actores locales y el perfil de su entorno; relaciona necesidades y actitudes humanas con los recursos naturales disponibles, situando al sujeto como actor preponderante en la búsqueda del progreso y evolución personal, familiar y comunitario, diferenciando al segmento asistencial del segmento promovible.

Para proceder se eligió una comunidad con exiguos recursos naturales, con pocos habitantes y, por consecuencia, desdeñada por su escasa aportación electoral. En ella se realizó la investigación mixta, que facilitara identificar las cualidades tanto de la población como de sus recursos y cuantificar los intereses de la población así como los mismos recursos. En atención a la normatividad de la institución de filiación, el universo se estratificó en menores y mayores de 16 años, seleccionando a este último como población de estudio. La información se examinó con software para análisis de datos.

\section{ESTRATEGIAS METODOLÓGICAS O MATERIALES Y MÉTODOS}

Se consultaron las declaraciones de las asambleas de la ONU y UNESCO, las publicaciones de especialistas en temas de desarrollo rural, desarrollo humano, formación de productores rurales, metodología de investigación, holismo, espiritualidad, valores morales, desarrollo humano, y desarrollo económico. Se procedió al análisis de contenido, triangulación de conceptos y construcción conceptual.

Siguiendo a Hernández (2015), se estratificó a los habitantes en mayores y menores de edad. Sin embargo, se careció de información oficial debido a que esa comunidad no se encontraba registrada en el Instituto Nacional de Estadística Geografía e Informática 
(INEGI). Por tal motivo, inicialmente se entrevistó al encargado local del cobro de agua potable, quien lleva el registro de tomas de agua domiciliarias, correspondiendo a una por familia; posteriormente se consultó al Director de la Escuela Primaria José María Morelos, para complementar la información.

Al número total de habitantes, se le restó la cantidad de menores de 16 años para determinar la unidad de análisis, a la cual se aplicó el factor propuesto por (Hernández (2005), de 0.2534, pág. 181, calculando así la muestra estadística. Mediante la distribución de frecuencias se identificó, entre los entrevistados, a los hombres y mujeres de menor y mayor edad. Con la mediana se determinó la edad intermedia por sexo y edad, y con la moda se identificó la mayor frecuencia también por sexo y edad. Se establecieron: categorías de datos tangibles y otra de datos sensibles, así como tres variables:

1. Desarrollo humano, con las siguientes subvariables: empoderamiento, democracia, valores cívicos y, resiliencia. En las primeras tres subvariables, los instrumentos de entrevista contenían opciones de respuesta múltiples, en escala tipo Likert, redactadas en términos comprensibles tanto coloquial como formalmente, esas opciones fueron: 1 . De plano nunca, 2. Casi nunca, 3. Ni si y Ni no, 4. Casi siempre, y 5. De plano siempre. La subvariable resiliencia, en concordancia con su condición, se indagó mediante preguntas abiertas, que se presentan en el siguiente orden: ¿qué prácticas productivas le gustaría mejorar? Nuevas actividades productivas que le gustaría emprender, ¿que actividades recreativas le gustaría practicar? ¿cuáles son sus habilidades sobresalientes? ¿qué conocimientos o experiencias le gustaría compartir con otras personas de la comunidad? ¿qué conocimientos o experiencias le gustaría que le compartan otras personas de la comunidad? ¿cuáles son sus aspiraciones personales? Estos instrumentos se sometieron a validación, aplicándolos inicialmente en la comunidad de San Antonio Corrales, considerando las similitudes en comunicación y costumbres con Nuevo Coronillas.

Para valorar las subvariables: empoderamiento, democracia y valores cívicos se estableció el criterio de niveles como: excelente, bueno, regular, deficiente y malo, que se determinaron a partir del cálculo porcentual de las cinco opciones de respuesta señaladas en el párrafo anterior, y que se asignaron en la escala de 80.01 a 100\%, 60.01 a $80 \%, 40.01$ a $60 \%, 20.01$ a $40 \%$, y 0 a $20.00 \%$. Ejemplificando lo anterior, la 
participación de la muestra en las decisiones familiares, fue del 76.32\%, ubicándose en el nivel bueno, por estar en la escala de 60.01 a $80 \%$.

Y para interpretar la subvariable empoderamiento, por tratarse de ítems de carácter abierto se procesaron con apoyo de software para análisis cualitativo, iniciando con la sistematización, de la cual surgieron códigos que se organizaron en siete familias, enseguida, en cada familia los códigos se subclasificaron en nodos, de acuerdo con las categorías emergentes de las respuestas obtenidas en las entrevistas, verificando la congruencia en el agrupamiento, pasando a la creación de redes semánticas, que ilustran el tipo de relación que los vincula.

2. Desarrollo económico. Esta variable se subdividió en las subvariables: Prácticas productivas que le gustaría mejorar y Nuevas prácticas productivas que le gustaría emprender. Los ítems, facilitaron respuestas de carácter abiertas y con la información obtenida, se estudiaron los datos sobre los bienes esenciales para la producción agropecuaria. En concordancia, se verificó esa información examinando la carpeta básica del comisariado y explorando la dotación ejidal, a fin de identificar la idoneidad productiva, mediante análisis empírico de las coloraciones y texturas predominantes en los suelos agrícolas, la disponibilidad y calidad del agua para su riego, así como sus prácticas productivas tradicionales.

\section{Gestión comunitaria: con las subvariables:}

Con el propósito de conocer distintos enfoques de la interpretación de la realidad, se entrevistó a los actores que representan las organizaciones sociales locales, iniciando con el Encargado del Orden, por ser la máxima autoridad en la comunidad.

Para realizar las entrevistas, se recorrieron las seis calles de la comunidad, visitando cada domicilio y procediendo a entrevistar al individuo que apareció primero. Por otro lado, se determinó una muestra no probabilística (Hernández, 2015), seleccionando a los ciudadanos que ocupaban cargos de representación en la organización interna de la comunidad, pág. 171, con la finalidad de obtener información general. Se resalta, que, tanto en la muestra probabilística como en la no probabilística, en cada entrevista se asistió personalmente al interrogado. Los datos obtenidos fueron examinados con apoyo de software.

Los ítems de carácter abierto se procesaron con apoyo de software para análisis cualitativo, iniciando con la sistematización, codificación y organización en familias de 


\section{El desarrollo comunitario}

datos, en las cuales se descubrieron categorías emergentes que posteriormente se subclasificaron en nodos. Enseguida se verificó la congruencia en el agrupamiento y finalmente se crearon redes semánticas que ilustran el tipo de relación que vincula los códigos. Es menester resaltar que en el proceso de estudio se tuvo presente la diferenciación entre desarrollo y crecimiento, así como la de desarrollo exógeno y endógeno.

\section{RESULTADOS Y DISCUSIÓN}

La comunidad contaba con 225 habitantes, de los cuales 77 eran menores de 16 años, de éstos, 15 aún no estaban en edad escolar, 11 eran alumnos de preescolar, 29 de educación primaria, dos estudiaban la secundaria, dos el bachillerato y 18 no estudiaban. La unidad de análisis quedó integrada con 148 individuos mayores de 16 años, la muestra resultó ser de 38 individuos.

Los datos examinados arrojaron el 100\% de casos válidos; la distribución de frecuencias indicó que en las entrevistas, los hombres de menor y mayor edad tenían 16 y 84 años respectivamente; en cambio, la mujer de menor edad contaba con 27 años y la mayor con 48. La mediana se determinó en 34 años, en ella se encontraron dos datos: la posición doce que registró 2 hombres y 2 mujeres, de 33 años cada uno, y la posición 13 que registró una mujer de 35 años; en cambio la moda, identificó a 3 hombres de 22 años. Los datos válidos acumulados fueron del $100 \%$.

Por otro lado, en cuanto al procesamiento de las variables, en lo correspondiente a desarrollo humano, se establecieron cuatro subdivisiones: empoderamiento, democracia, valores cívicos, y resiliencia. En las primeras tres subvariables, las opciones de respuesta fueron múltiples, en escala tipo Likert, que favorecieron la valoración del ánimo con que se contestaron los ítems; y se redactaron en términos comprensibles tanto coloquial como formalmente, esas opciones fueron: 1. De plano nunca, 2. Casi nunca, 3. Ni si y Ni no, 4. Casi siempre, y 5. De plano siempre.

En concordancia, para valorar las subvariables mencionadas, se estableció el criterio de niveles como: excelente, bueno, regular, deficiente y malo, que se determinaron a partir del cálculo porcentual de las cinco opciones de respuesta señaladas en el párrafo anterior, y que se asignaron en la escala de 80.01 a 100\%, 60.01 a $80 \%, 40.01$ a 60\%, 20.01 a 40\%, y 0 a $20.00 \%$. Ejemplificando lo anterior, la participación de la muestra en las decisiones familiares, fue del $76.32 \%$, ubicándose en el nivel bueno; sin embargo, el promedio 
porcentual que se obtuvo de las respuestas a los cinco ítems de la subvariable empoderamiento fue de 73.68 , que se ubicó en la escala de 60.01 a $80 \%$, y a la cual se le asignó el criterio de buen nivel de empoderamiento. En tal sentido, enseguida se describen, los datos recuperados en campo.

\subsection{Descripción de datos de campo, sensibles y tangibles}

\section{- Datos de campo sensibles}

En la subvariable empoderamiento, se planteó: A. ¿Participa en las decisiones familiares?, B. Participa en la toma de decisiones de la comunidad?, C. ¿Es resistente a las acciones discriminatorias?, D. Sus familiares y amigos ¿Le solicitan ayuda?, E. Los candidatos a cargos públicos ¿Le piden apoyo?, en todos los casos se obtuvo 100\% de datos válidos. A la pregunta ¿Participa en las decisiones familiares?, en el segmento de 16 a 25 años, la pregunta "ni sí y ni no" fue la opción de respuesta de un hombre, "casi siempre", fue la elección de cuatro hombres, y "de plano siempre" fue escogida por tres hombres; luego, en el intervalo de 26 a 35 años la respuesta "de planos nunca" fue elegida por un hombre y una mujer, "casi nunca" fue la opción de dos mujeres, "ni sí ni no" la prefirieron dos hombres, "casi siempre" fue preferida por cuatro hombres y seis mujeres, "de plano siempre" la prefirió uno hombre y dos mujeres; en el intervalo de 46 a 55 años, la respuesta "casi nunca" fue preferida por un hombre, "ni sí ni no" también la prefirió un hombre, "de plano siempre" la prefirió una mujer, en este intervalo se obtuvieron respuestas de dos hombres y una mujer; en el intervalo de 56 a 65 años la respuesta "casi siempre" fue la opción; en el intervalo de 66 a 75 años como "casi siempre" contestaron dos hombres, " de plano siempre" fue la respuesta de un hombre, en suma se obtuvieron tres respuestas; y en el intervalo de 76 a 85 años y más, como “de plano siempre” contestó un hombre. Se obtuvieron respuestas de 25 hombres y trece mujeres.

Correspondiente al ítem ¿participa en la toma de decisiones de la comunidad?, En el intervalo de dieciséis a 25 años contestaron como "casi nunca" tres hombres, "ni sí y ni no" dos hombres, "casi siempre" los hombres, en suma ocho respuestas; en el intervalo de 26 a 35 años en contestaron "de plano nunca" dos mujeres," casi nunca" tres hombres, "ni sí y ni no" cinco mujeres, "casi siempre" un hombre y cuatro mujeres "de plano siempre" cuatro hombres, ocho hombres y once mujeres; en el intervalo de 36 a 45 años contestaron "casi nunca" un hombre, "casi siempre" una mujer, en suma dos respuestas; en el intervalo de 46 a 55 años en contestaron "casi siempre", un hombre y una mujer, 
"de plano siempre" un hombre, en suma dos hombres y una mujer; en cuanto al intervalo de 56 a 65 años dos hombres contestaron "casi siempre"; en el intervalo de 66 a 75 años como "casi siempre" contestaron dos hombres y como "de plano siempre" contestó un hombre, en suma tres respuestas de hombres; y en el intervalo de 76 a 85 años y más un hombre prefirió como respuesta "de plano siempre". En total se obtienen 38 datos válidos, que corresponden a 25 hombres y trece mujeres.

Respecto a ¿es resistente a las acciones discriminatorias? en el segmento de 16 a 25 años, la respuesta "casi nunca" fue preferida por un hombre, "ni si y ni no" fue la opción de cinco hombres, "casi siempre" fue la opción de dos hombres, en suma contestaron ocho hombres; en el intervalo de 26 a 35 años contestaron como "de plano nunca" dos hombres y dos mujeres, "casi nunca" dos hombres y tres mujeres, "ni si y ni no" un hombre y una mujer, "casi siempre" tres hombres y cuatro mujeres, "de plano siempre" únicamente una mujer, en este intervalo en suma se obtuvieron respuestas de ocho hombres y once mujeres; en el intervalo de 36 a 45 años un hombre prefirió como respuesta "ni sí y ni no" y una mujer "de plano siempre", sumando dos respuestas; en el intervalo de 46 a 55 años "de plano nunca" fue la respuesta de un hombre, "casi nunca" fue preferida por una mujer, "de plano siempre" fue elegida por un hombre, en suma se obtuvieron respuestas de dos hombres y una mujer; en el intervalo de 56 a 65 años, un hombre contestó "de plano nunca", y otro "casi nunca", que suman dos respuestas; en el intervalo de 66 a 75 años, un hombre contestó "de plano nunca", otro "casi nunca", y otro más "casi siempre”, que suman respuestas de tres hombres; y en el intervalo de 76 a 85 años y más, un hombre eligió como respuesta “de plano nunca”. En este ítem, también se obtienen 38 datos válidos, de 25 hombres y trece mujeres.

En las incidencias de la pregunta "sus familiares y amigos ¿le solicitan ayuda?”, resultó que en el intervalo de 16 a 25 años, un hombre contestó "de planos nunca", otro "ni sí y ni no", cinco "casi siempre", y uno más "de plano siempre", en este intervalo se obtuvieron respuestas de ocho hombres; en el intervalo de 26 a 35 años, "casi nunca" fue la respuesta de un hombre y una mujer, "ni si y ni no" fue la opción de dos hombres y dos mujeres, "casi siempre" fue elegida por cinco hombres y siete mujeres, y "de plano siempre" la prefirió una mujer, en este intervalo se obtuvieron respuestas de ocho hombres y once mujeres. En el intervalo de 36 a 45 años una mujer optó por la respuesta "de plano nunca", y un hombre eligió "casi siempre"; en el intervalo de 46 a 55 años dos 
hombres y una mujer contestaron "casi siempre", sumando tres respuestas; en el intervalo de 56 a 65 años un hombre contestó un "casi siempre", y otro eligió "de plano siempre", sumando dos respuestas; en el intervalo de 66 a 75 años un hombre prefirió "de plano nunca", y dos “casi siempre"; y en el intervalo de 76 a 85 años y más un hombre contestó “de plano siempre”. Se obtuvieron 38 datos válidos, de 25 hombres y 13 mujeres.

En la pregunta "los candidatos a cargos públicos ¿le piden apoyo? en el intervalo de 16 a 25 años la respuesta "de plano nunca", fue elegida por dos hombres, "casi nunca" por uno, "ni sí y ni no" por uno, "casi siempre" por dos, y "de plano siempre” por otros dos, sumando ocho respuestas; en el intervalo de 26 a 35 años, dos hombres eligieron como respuesta "de plano nunca", un hombre y tres mujeres prefirieron "casi nunca", un hombre y dos mujeres "ni sí y ni no", tres hombres y cuatro mujeres "casi siempre”, un hombre y dos mujeres "de plano siempre", sumando diecinueve respuestas de ocho hombres y once mujeres; en el intervalo de 36 a 45 años un hombre prefirió la respuesta "casi siempre", y una mujer eligió "de plano siempre"; en el intervalo de 46 a 55 años un hombre y una mujer optaron por contestar "casi siempre", y un hombre prefirió como respuesta "de plano siempre", sumando tres respuestas; en el intervalo de 56 a 65 años, un hombre contestó "casi siempre", y otro prefirió "de plano siempre", sumando dos respuestas; en el intervalo de 66 a 75 años, un hombre prefirió como respuesta "casi nunca”, y dos más optaron por "de plano siempre", sumando tres respuestas; y en el intervalo de 76 a 85 años y más, un hombre contestó “de plano siempre”. En resumen, se obtienen 38 datos válidos, de 25 hombres y trece mujeres.

Cuadro 1. Concentrado de la subvariable empoderamiento.

\begin{tabular}{|l|c|c|c|c|}
\hline \multicolumn{1}{|c|}{ IItems } & \multicolumn{2}{c|}{ Hombres } & \multicolumn{2}{c|}{ Mujeres } \\
\hline Nivel de participación en las decisiones familiares & 19 & 50.00 & 10 & 26.32 \\
\hline $\begin{array}{l}\text { Nivel de participación en la toma de decisiones de } \\
\text { la comunidad }\end{array}$ & 16 & 42.11 & 6 & 15.79 \\
\hline Nivel de resistencia a las acciones discriminatorias & 7 & 18.42 & 6 & 15.79 \\
\hline Nivel de solicitud de ayuda, de familiares y amigos & 19 & 50.00 & 9 & 23.68 \\
\hline $\begin{array}{l}\text { Nivel de solicitud de apoyo, de candidatos a cargos } \\
\text { públicos }\end{array}$ & 16 & 42.11 & 8 & 21.05 \\
\hline Porcentaje de empoderamiento por género & \multicolumn{3}{|c|}{40.53} & \multicolumn{3}{|c|}{20.53} \\
\hline Nivel, porcentual, de empoderamiento & \multicolumn{5}{|c|}{} \\
\hline
\end{tabular}

En cuanto a la subvariable democracia a "en su hogar, para tomar decisiones ¿usted consulta a sus familiares?", en el intervalo de 16 a 25 años, cuatro hombres y tres mujeres 
contestaron "casi nunca", un hombre "ni si y ni no", tres más "casi siempre", y otros dos "de plano siempre", sumando 13 respuestas de 10 hombres y tres mujeres; en el intervalo de 26 a 35 años, un hombre contestó “de plano nunca”, uno más y una mujer contestaron "ni si y ni no", dos hombres y cuatro mujeres optaron por "casi siempre", tres hombres y dos mujeres prefirieron "de plano siempre", sumando 14 respuestas de seis hombres y ocho mujeres; en el intervalo de 36 a 45 años un hombre contestó "casi siempre", y una mujer "de plano siempre", sumando 2 respuestas; en el intervalo de 46 a 55 años, una mujer contestó "casi siempre", y dos hombres optaron por "de plano siempre", sumando tres respuestas; en el intervalo de 56 a 65 años, un hombre contestó "casi siempre", y otro "de plano siempre", sumando dos respuestas; en el intervalo de 66 a 75 años, tres hombres contestaron "de plano siempre"; y en el intervalo de 76 a 85 años y más, un hombre contestó “de plano siempre”. Resumiendo, se obtuvieron 25 respuestas de hombres y 13 de mujeres, totalizando 38 datos válidos.

A la pregunta: en la comunidad, para tomar decisiones ¿usted consulta a los habitantes? Un hombre de 16 a 25 escogió como respuesta "de plano nunca", dos "casi nunca", dos más "ni si y ni no", y tres "casi siempre", sumando ocho respuestas de hombres; en el intervalo de 26 a 35 años, un hombre y cuatro mujeres contestaron "casi nunca", un hombre y una mujer "ni sí y ni no", cuatro hombres y cinco mujeres optaron por "casi siempre", y como "de plano siempre" se decidieron dos hombres y una mujer, sumando 19 respuestas de ocho hombres y 11 mujeres; en el intervalo de 36 a 45 años un hombre y una mujer respondieron "de plano siempre", sumando dos respuestas; en el intervalo de 46 a 55 años, un hombre contestó "ni sí y ni no", una mujer prefirió contestar "casi siempre", y otro más se decidió por "de plano siempre", sumando tres respuestas de dos hombres y una mujer; en el intervalo de 56 a 65 años, dos hombres prefirieron "casi siempre"; en el intervalo de 66 a 75 años, un hombre contestó "ni sí y ni no", y dos más prefirieron "de plano siempre", sumando tres respuestas; y en el intervalo de 76 a 85 años y más, un hombre eligió la respuesta "de plano siempre". En resumen se obtuvieron respuestas de 25 hombres y trece mujeres, y en total 38 datos válidos.

Respecto a la subvariable Democracia, referente a la pregunta ¿Le agrada aceptar cargos públicos?, en el intervalo de 16 a 25 años, la respuesta "de planos nunca" fue la opción de un hombre, "casi nunca" fue elegida por otro, "ni sí y ni no" por cuatro, "casi siempre" fue la decisión de uno más, y "de plano siempre” de otro, sumando ocho respuestas; en el 
intervalo de 26 a 35 años, dos hombres y cuatro mujeres eligieron como respuesta "de plano nunca", dos hombres y dos mujeres contestaron "casi nunca", dos hombres y dos mujeres se inclinaron por "ni sí y ni no", dos hombres por "casi siempre”, dos hombres y una mujer por "de plano siempre”, sumando 19 respuestas de ocho hombres y 11 mujeres; en el intervalo de 36 a 45 años, una mujer contestó “casi nunca", y un hombre contestó “ni sí y ni no", sumando dos respuestas; en el intervalo de 46 a 55 años, un hombre eligió como respuesta "casi nunca", una mujer decidió "casi siempre", y un hombre optó por "de plano siempre", sumando tres respuestas de dos hombres y una mujer; en el intervalo de 56 a 65 años, dos hombres contestaron "casi siempre"; en el intervalo de 66 a 75 años, un hombre contestó "de plano nunca", otro "casi siempre”, y otro más "de plano siempre", sumando tres respuestas; en el intervalo de 76 a 85 años y más, un hombre eligió la respuesta "de plano siempre". Resumiendo, se obtuvieron 25 respuestas de hombres y trece de mujeres, que totalizan 38 datos válidos.

\section{Cuadro 2. Concentrado de la subvariable democracia.}

\begin{tabular}{|l|c|c|c|c|}
\hline \multicolumn{1}{|c|}{ Ítems } & \multicolumn{2}{|c|}{ Hombres } & Mujeres \\
\hline $\begin{array}{l}\text { En su hogar, para tomar decisiones ¿Usted consulta a sus } \\
\text { familiares? }\end{array}$ & 19 & 50.00 & 8 & 21.05 \\
\hline $\begin{array}{l}\text { En la comunidad, para tomar decisiones ¿Usted consulta } \\
\text { a los habitantes? }\end{array}$ & 16 & 42.11 & 8 & 21.05 \\
\hline ¿Le agrada aceptar cargos públicos? & 10 & 26.32 & 4 & 10.53 \\
\hline Nivel, porcentual, de democracia, por género & & 39.48 & & 17.52 \\
\hline Nivel, porcentual, de democracia en la comunidad & \multicolumn{4}{|c|}{$\mathbf{5 7 . 0 0}$} \\
\hline
\end{tabular}

La subvariable valores cívicos, que también tiene $100 \%$ de casos válidos, pregunta: A. ¿Respeta los símbolos patrios?, B. Cuando se entera de algún abuso ¿Lo denuncia?, C. ¿Opina sobre la situación del país?, D. ¿Opina sobre los gobernantes?, y las respuestas correspondientes se analizan por ítem, intervalo de edades, y género de los entrevistados. En las siguientes líneas se detallan estos datos.

En cuanto a ¿respeta los símbolos patrios?, en el intervalo de 16 a 25 años, la respuesta "de plano nunca" fue elegida por un hombre, "casi siempre" por tres hombres, "de plano siempre" otros, sumando ocho respuestas; en el intervalo de 26 a 35 años un hombre eligió como respuesta "de plano nunca", otro prefirió "casi nunca", dos hombres y cinco mujeres se decidieron por "casi siempre", cuatro hombres y seis mujeres optaron por "de plano siempre", sumando 19 respuestas de ocho hombres y 11 mujeres; en el intervalo de 
36 a 45 años un hombre eligió "de plano siempre"; en el intervalo de 46 a 55 años, una mujer contestó "casi siempre", y dos hombres respondieron "de plano siempre", sumando tres respuestas; en el intervalo de 56 a 65 años un hombre eligió como respuesta "casi siempre", y otro prefirió "de plano siempre", sumando dos respuestas; en el intervalo de 66 a 75 años, un hombre contestó "casi siempre", y otros dos "de plano siempre", sumando tres respuestas; en el intervalo de 76 a 85 años y más, un hombre contestó "de plano siempre". En resumen, se tienen 25 respuestas de hombres y trece mujeres, con un total de 38 datos válidos.

A la pregunta: cuando se entera de algún abuso ¿Lo denuncia? en el intervalo de 16 a 25 años, dos hombres contestaron "casi nunca", un hombre prefirió "ni sí y ni no”, cuatro "casi siempre”, y uno hombre decidió “de plano siempre", sumando ocho respuestas; en el intervalo de 26 a 35 años, un hombre y dos mujeres contestaron "de plano nunca", tres hombres y una mujer eligieron "casi nunca", tres hombres y cuatro mujeres se inclinaron por "ni si y ni no", tres mujeres optaron por "casi siempre", un hombre y una mujer eligieron "de plano siempre", obteniéndose respuestas de ocho hombres y 11 mujeres para una suma de 19; en el intervalo de 36 a 45 años, un hombre contestó "de plano nunca", una mujer eligió "ni sí y ni no", y un hombre contestó un "casi siempre”, sumando tres respuestas de un hombre y dos mujeres; en el intervalo de 46 a 55 años, un hombre contestó "ni si y ni no", y otro prefirió “de plano siempre”, sumando dos respuestas; en el intervalo de 56 a 65 años, un hombre eligió "casi siempre", y otro decidió "de plano siempre", sumando dos respuestas; en el intervalo de 66 a 75 años, dos hombres optaron por "casi siempre", y otro eligió "de plano siempre", sumando tres respuestas; en el intervalo de 76 a 85 años y más, un hombre optó “de plano siempre”. El resumen indica que respondieron 25 hombres y trece mujeres, con un total de 38 datos válidos.

En la pregunta ¿Opina sobre la situación actual del país?, En el intervalo de 16 a 25 años, dos hombres contestaron "de plano nunca", uno contestó "casi nunca", dos prefirieron "ni sí y ni no", dos más "casi siempre”, y otro "de plano siempre”, sumando ocho respuestas; en el intervalo de 26 a 35 años, una mujer optó por "de plano nunca", tres hombres y dos mujeres por "casi nunca", dos hombres y cinco mujeres decidieron "ni sí y ni no ", dos hombres y dos mujeres optaron por "casi siempre", y un hombre y una mujer por "de plano siempre", sumando respuestas de ocho hombres y once mujeres; en el intervalo de 36 a 45 años, una mujer contestó "de plano nunca", y un hombre decidió "ni sí y ni no", 
sumando dos respuestas; en el intervalo de 46 a 55 años, un hombre optó por contestar “de plano nunca”, una mujer por "ni si y ni no", y un hombre por "casi siempre”, sumando tres respuestas de dos hombres y una mujer; en el intervalo de 56 a 65 años, dos hombres eligieron "casi siempre"; en el intervalo de 66 a 75 años, un hombre optó por "casi siempre", y otros dos prefirieron "de plano siempre", sumando tres respuestas; y en el intervalo de 76 a 85 años y más, un hombre eligió la respuesta "de plano siempre". Resumiendo, se obtuvieron respuestas de 25 hombres y trece mujeres, y un total de 38 datos válidos.

En la última pregunta: ¿opina sobre los gobernantes?, en el segmento de 16 a 25 años, cuatro hombres contestaron "casi nunca", dos eligieron "ni si y ni no", y dos más optaron por "casi siempre", sumando ocho datos; en el intervalo de 26 a 35 años, la respuesta "de plano nunca", fue preferida por dos hombres, "casi nunca" de dos hombres y dos mujeres, "ni si y ni no" por dos hombres y tres mujeres, "casi siempre" por un hombre y cinco mujeres, "de plano siempre" por un hombre y una mujer, obteniendo ocho respuestas de hombres que suman diecinueve respuestas; en el intervalo de 36 a 45 años, la respuesta "de planos nunca" la prefirió una mujer, y "casi nunca" la prefirió un hombre, sumando dos respuestas en el intervalo de 46 a 55 años, "de plano nunca" fue la respuesta de un hombre, "ni si y ni no" contestó una mujer, "casi siempre" respondió un hombre, sumando tres respuestas de dos hombres y una mujer; en el intervalo de 56 a 65 años, de plano nunca contestó un hombre, y otro optó por "casi nunca" sumando dos respuestas; en el intervalo de 66 a 75 años, "casi siempre" fue la preferencia de un hombre, y "de plano siempre" sumando tres respuestas; y en el intervalo de 76 a 85 años y más "de plano siempre" fue la respuesta de un hombre. Resumiendo, se obtuvieron respuestas de 25 hombres y trece mujeres, totalizando 38 datos válidos.

\section{Cuadro 3. Concentrado de la subvariable civismo}

\begin{tabular}{|l|c|c|c|c|}
\hline \multicolumn{1}{|c|}{ Resumen de civismoms } & \multicolumn{2}{|c|}{ Hombres } & \multicolumn{2}{c|}{ Mujeres } \\
\hline ¿Respeta los símbolos patrios? & 22 & 57.89 & 13 & 34.21 \\
\hline Cuando se entera de algún abuso ¿Lo denuncia? & 14 & 36.84 & 4 & 10.53 \\
\hline ¿Opina sobre la situación actual del país? & 13 & 34.21 & 3 & 7.89 \\
\hline ¿Opina sobre los gobernantes? & 9 & 23.68 & 6 & 15.79 \\
\hline Nivel, porcentual, cívico, por género & \multicolumn{5}{|c|}{38.16} & \multicolumn{3}{|c|}{17.11} \\
\hline Nivel, porcentual, de valores cívicos, en la comunidad & \multicolumn{5}{|c|}{} \\
\hline
\end{tabular}


Con las medidas porcentuales anteriores, se deduce un valor medio. Ver cuadro 4.

Cuadro 4. Síntesis de las subvariables empoderamiento, democracia y valores cívicos, en el nivel comunitario.

\begin{tabular}{|l|c|c|c|}
\hline \multirow{2}{*}{ Súntesis } & \multicolumn{3}{|c|}{ Porcentajes } \\
\cline { 2 - 4 } & Hombres & Mujeres & Nivel comunitario \\
\hline Empoderamiento & 40.53 & 20.53 & 61.06 \\
\hline Democracia & 39.48 & 17.52 & 57.00 \\
\hline Valores cívicos & 38.16 & 17.11 & 55.26 \\
\hline Promedio & $\mathbf{3 9 . 3 9}$ & $\mathbf{1 8 . 3 9}$ & $\mathbf{5 7 . 7 7}$ \\
\hline
\end{tabular}

La última sub-variable, resiliencia, en concordancia con su condición, se indaga mediante preguntas abiertas, que se presentan en el siguiente orden: ¿qué prácticas productivas le gustaría mejorar? Nuevas actividades productivas que le gustaría emprender, que actividades recreativas le gustaría practicar, cuáles son sus habilidades sobresalientes, qué conocimientos o experiencias le gustaría compartir con otras personas de la comunidad, qué conocimientos o experiencias le gustaría que le compartan otras personas de la comunidad, cuáles son sus aspiraciones personales. El resumen de casos, de su análisis estadístico, indica que los instrumentos de entrevistas se aplicaron al 100\% de la muestra. En cuanto al análisis por ítem, en las incidencias a la pregunta ¿Qué prácticas productivas le gustaría mejorar?, en el intervalo de 16 a 25 años, seis hombres si contestaron, y dos no contestaron; en el intervalo de 26 a 35 años contestaron seis hombres y cinco mujeres, y no contestaron otros dos hombres y seis mujeres, sumando en este intervalo 11 que si contestaron y ocho que no contestaron; en el intervalo de 36 a 45 años, sí contestaron un hombre y una mujer, sumando dos sujetos; en el intervalo en de 46 a 55 años, si contestaron dos hombres y una mujer; en el intervalo de 56 a 65 años, si contestaron dos hombres; en el intervalo de 66 a 75 años, si contestaron tres hombres; y en el intervalo de 76 a 85 años y más, si contestó un hombre; en resumen: los hombres sí contestaron 21 veces, y no contestaron en cuatro ocasiones; y la mujeres si contestaron siete veces y no contestaron en 6 ; sintetizando, contestaron 28 y 10 no contestaron; en total se obtuvieron 38 datos válidos.

Referente a nuevas actividades productivas que le gustaría emprender, en el intervalo de 16 a 25 años, si contestaron cinco hombres, y no contestaron tres hombres; en el intervalo 
de 26 a 35 años, si contestaron cuatro hombres y siete mujeres, no contestaron cuatro hombres y cuatro mujeres, en suma si contestaron once entrevistados y no contestaron ocho; en el intervalo de 36 a 45 años, sí contestaron un hombre y una mujer, en suma, si contestaron dos personas; en el intervalo de 46 a 55 años, si contestaron dos hombres y una mujer, en suma, si contestaron tres personas; luego, en el intervalo de 56 a 65 años, si contestó un hombre, y no contestó otro hombre; en cuanto al intervalo de 66 a 75 años, si contestaron tres hombres; y en el intervalo de 76 a 85 años y más, si contestó un hombre; resumiendo, si contestaron 17 hombres y nueve mujeres, sumando 26 personas, y no contestaron ocho hombres y cuatro mujeres sumando 12 personas; en total se obtienen 38 datos válidos.

En cuanto a ¿Qué actividades recreativas le gustaría practicar?, en el intervalo de 16 a 25 años, si contestaron ocho hombres; en el intervalo de 26 a 35 años sí contestaron ocho hombres y once mujeres, sumando diecinueve respuestas; en el intervalo de 36 a 45 años, si contestó un hombre y una mujer, sumando dos respuestas; en el intervalo de 46 a 55 años, sí contestaron dos hombres y una mujer, sumando tres respuestas; en el intervalo de 56 a 65 años, sí contestaron dos hombres; en el intervalo de 66 a 75 años, sí contestaron tres hombres; y en el intervalo de 76 a 85 años y más, sí contestó un hombre; en resumen, sí contestaron 25 hombres y trece mujeres, totalizando 38 datos válidos.

Respecto a cuáles son sus habilidades sobresalientes, se evidencia que en el intervalo de dieciséis a 25 años, sí contestaron siete hombres, y uno no contestó; en el intervalo de 26 a 35 años sí contestaron seis hombres y 11 mujeres, sumando 17 personas que si contestaron, y no contestaron dos hombres; en el intervalo de 36 a 45 años, si contestó una mujer, y no contestó un hombre; en el intervalo de 46 a 55 años, si contestaron dos hombres y una mujer, que suman tres respuestas; en el intervalo de 56 a 65 años, si contestaron dos hombres; en el intervalo de 66 a 75 años, si contestaron tres hombres; en el intervalo de 76 a 85 años y más, si contestó un hombre; el resumen indica que si contestaron 20 hombres y 13 mujeres, que suman 33 respuestas, y no contestaron 5 hombres, en total se cuenta con 38 datos válidos.

Respecto a ¿qué conocimientos o experiencias le gustaría compartir?, en el intervalo de 16 a 25 años, si contestaron ocho hombres; en el intervalo de 26 a 35 años, si contestaron cinco hombres y 10 mujeres, no contestaron tres hombres y una mujer, en suma, sí contestaron 15 personas, y no contestaron cuatro personas; en el intervalo de 36 a 45 años, 
si contestó una mujer y no contestó un hombre; en el intervalo de 46 a 55 años, si contestaron un hombre y una mujer, y no contestó un hombre, en suma, si contestaron dos personas, y no contestó una persona; en el intervalo de 56 a 65 años, si contestó un hombre, y no contestó otro hombre; en el intervalo de 66 a 75 años, si contestaron tres hombres; en el intervalo de 76 a 85 años y más, si contestó un hombre; el resumen muestra que si contestaron 18 hombres y 12 mujeres, sumando 30 personas, y no contestaron siete hombres y una mujer, sumando ocho personas, que totalizan 38 datos válidos.

Continuando, en el ítem ¿qué conocimientos o experiencias le gustaría que le compartan otras personas de la comunidad?, en el intervalo de 16 a 25 años, siete hombres si contestaron, y uno no contestó; en el intervalo de 26 a 35 años, seis hombres y 10 mujeres si contestaron, y dos hombres y una mujer no contestaron, en suma 16 entrevistados si contestaron y tres no contestaron; en el intervalo de 36 a 45 años, un hombre si contestó y una mujer no contestó; en el intervalo de 46 a 55 años, un hombre y una mujer si contestaron, y un hombre no contesto, en suma si contestaron dos personas y otra no contestó; en el intervalo de 56 a 65 años, si contestaron dos hombres; en el intervalo de 66 a 75 años, si contestaron tres hombres; y en el intervalo de 76 a 85 años y más, si contestó un hombre; en resumen, si contestaron 20 hombres y 11 mujeres, que suman 31 personas, y no contestaron cinco hombres y dos mujeres, que suman siete personas, totalizando 38 datos válidos.

Respecto al último ítem, ¿cuáles son sus aspiraciones personales?, en el intervalo de 16 a 25 años, si contestaron ocho hombres; en el intervalo de 26 a 35 años, si contestaron seis hombres y ocho mujeres, y no contestaron dos hombres y tres mujeres, en suma, si contestaron catorce personas y no contestaron cinco personas; en el intervalo de 36 a 45 años, si contestaron un hombre y una mujer, en suma dos personas; en el intervalo de 46 a 55 años, si contestaron un hombre y una mujer, y no contestó un hombre, en suma dos personas si contestaron y una no contestó; en el intervalo de 56 a 65 años, si contestó un hombre y no contestó una mujer; en el intervalo de 66 a 75 años, si contestaron dos hombres, y uno no contestó; y en el intervalo de 76 a 85 años y más, si contestó un hombre; en resumen, si contestaron 19 hombres y diez mujeres, que suman 29 entrevistados, y no contestaron seis hombres y tres mujeres, que suman nueve personas, totalizando 38 datos válidos. Los valores anteriores, se sintetizan en el cuadro 5. 
Cuadro 5. Síntesis porcentual comunitario, de respuestas a los cuestionamientos sobre resiliencia.

\section{Síntesis}

\begin{tabular}{|l|c|c|c|}
\hline $\begin{array}{l}\text { Nivel comunitario, porcentual, de } \\
\text { resiliencia }\end{array}$ & Hombres & Mujeres & Resumen \\
\cline { 3 - 4 } & $53 \%$ & $28 \%$ & $81 \%$ \\
\hline
\end{tabular}

\section{- Descripción de datos tangibles}

En este apartado, se describen los datos sobre los bienes esenciales, para la producción agropecuaria. Éstos se recuperaron a partir de la información obtenida sobre las actividades productivas tradicionales, de la población de Nuevo Coronillas; en concordancia, se examinó la carpeta básica ejidal y se exploró su dotación ejidal, a fin de identificar las texturas predominantes en los suelos agrícolas, la disponibilidad de agua para su riego y sus prácticas productivas.

Derivado de lo anterior, se establece que el núcleo agrario usufructúa 55-00 hectáreas de labor, de las cuales 47-00 son de temporal y 8-00 de riego, así como 120-00 de agostadero, y 4-70 de zona urbana, sumando 170-70 Has. En la imagen 1, se muestra la vista aérea de la dotación ejidal, en ella se aprecia una porción con figuras rectangulares, que corresponden a las dotaciones ejidales individuales, mismas que son susceptibles de riego; también se ven varias figuras despejadas y delimitadas irregularmente, que corresponden a las parcelas de labor de temporal; y el resto es el área de agostadero.

En la imagen 1, se observa una fracción de la dotación ejidal, notándose la pendiente de los suelos de labor de temporal, y las tierras bajas, de labor de riego. Lo escarpado del terreno, ilustra sobre las dificultades para el tránsito de maquinaria agrícola, y la imposibilidad de acceder al caudal del Rio Grande, para regar los cultivos de maíz. Imagen 1. Suelos con pendiente de labor de temporal y tierras bajas de labor de riego

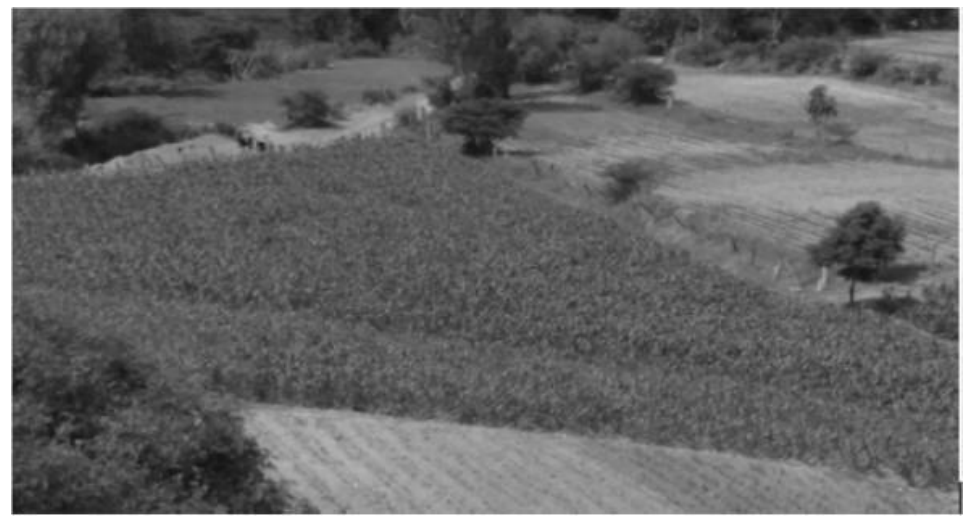


En cuanto a la textura, se testifica mediante examen empírico del suelo, estimándose que, en la composición de la tierra de labor, predomina la arcilla, en segundo lugar, la arena, y en tercero el limo, por tanto, se clasifica como arcillo-arenosa-limosa. Esto significa que tiene regular cualidad para retener la humedad, situación benéfica para las plantas, cuando deja de llover por periodos prolongados.

Por otro lado, se entrevistó a los representantes de las organizaciones sociales locales. Del Encargado del Orden, máxima autoridad en Nuevo Coronillas, se recupera la información que se transcribe enseguida:

- Queremos un apoyo para el Municipio de Nuevo Coronillas, queremos arreglen las calles y las canchas de fútbol y básquetbol, reparación del piso.

- Y los alumbrados del municipio de coronillas.

- Registrar la comunidad de Coronillas.

- Y no tirar basura en el municipio.

- Enseñar, a jóvenes y adultos, oficios.

Así mismo, se entrevista a los miembros del Comisariado Ejidal del Ejido Nuevo Coronillas; la información que proporcionaron, al igual que la anterior, también se transcribe a continuación:

- Semilla de pasto para el ganado.

- Balastro para los caminos.

- Material para la casa ejidal.

- Un toro semental para [pie de cría de ganado] de engorda.

- Probar semillas de maíz.

- Enseñar a los niños a sembrar.

- Intercambios con otros ejidos.

- Un pozo para riego o para la [sic, el] agua potable.

También, la Mesa Directiva de la Asociación de Padres de Familia, de la Escuela Primaria “José María Morelos”, hace el planteamiento siguiente:

- Tejado para el patio (lámina).

- Cancha de basquetbol.

- Pintura para la escuela.

- Cancha de fútbol.

- Reconstrucción de la malla que rodea la escuela [cerco perimetral]. 
- Alumbrado dentro de la escuela, ya que está muy obscuro por las noches.

- Impermeabilizante para los salones.

- Tejado para el lugar donde se encuentra la pila y un lavadero.

- Reparación del equipo de cómputo.

- Apoyo para el maestro, con otro docente.

- Talleres para los niños (donde enseñen algún oficio).

- Proyecto de ampliación de la escuela.

Igualmente, se consulta a la Dirección de la Escuela Primaria "José María Morelos", quien proporciona la información que se describe:

Necesidades prioritarias de la escuela:

- Dos pintarrones.

- Pintura para las aulas.

- Vidrios para las aulas.

- Ampliación para los baños.

- Cancha de basquetbol.

- Techo del patio cívico.

- Arreglo del cerco perimetral.

- El trazo de dos salones de clases.

- Una bodega para guardar los alimentos de la cocina.

- Registrar la comunidad con su nombre oficial.

- Un invernadero.

- Impermeabilizante.

- Ampliación de la escuela en dos aulas.

- Intercambio cultural.

- Internet.

- El cañón [proyector] no sirve.

- La impresora no sirve.

Por otro lado, la representante de la Capilla del Santo Niño de Praga expone:

- Reconstrucción de la capilla.

- Material para los cursos de catecismo: sillas, mesas, colores, pizarrones, acuarelas, lápiz, hojas blancas.

- Tejado. 
- Baño.

- Patio.

Los datos descritos aquí, después de su acopio en campo, y de su sistematización para concentrarlos, son el preámbulo del análisis que se realiza en el subcapítulo siguiente, con el propósito de que el autor, encuentre en ellos, su significado, para, posteriormente favorecer la oportunidad de acercarse a la realidad de la comunidad de Nuevo Coronillas, del Municipio de Charo.

\section{CONCLUSIÓN O CONSIDERACIONES FINALES}

Por tanto, y asumiendo que la intervención educativa es un recurso pedagógico, que desde la perspectiva educativa favorece la solución de problemas sociales, y que en el segmento de adultos guía la coparticipación de agentes externos y actores locales, en pro de la solución de la problemática sentida y real, mediante el trabajo colaborativo, se recuperan sustancialmente los referentes, en este sentido, de la ONU y la UNESCO. Por consiguiente, se resaltan las bondades de la intervención educativa, que como disciplina es el medio para desadormecer los valores de una sociedad en letargo que, obviamente, al reanimarse se volverá más razonable, crítica y demandante; pero que también favorece la rectificación de la interpretación torcida de los supuestos sociales, que halagan el bochorno de la farándula, la malvivencia y el crimen, que vician la industria, comercio, profesión o política, así como de los que encubiertos en gris refinamiento se ingenian para el hurto y desfalco.

Teóricamente esta propuesta, de intervención educativa, se posiciona epistemológicamente en el criticismo, en el ámbito de la educación no formal, en el humanismo y en el enfoque dual, por la conexión de estos conceptos con los productos emergentes del ejercicio de aproximación a la realidad, además empalma con la afirmación de Rogers (2007), sobre el hombre, a quien considera "digno de confianza, cuyas características más profundas tienden hacia el desarrollo, la diferenciación, las relaciones cooperativas..." (pág. 31).

Por tanto, la propuesta que aquí se plantea, pondera el carácter endógeno del desarrollo comunitario y sostiene que la intervención educativa, es elemento ineludible en la coparticipación institucional o privada cuando se pretende abonar al desarrollo. Así, pues, esta propuesta, geográficamente se circunscribe a la comunidad de Nuevo Coronillas, particularmente al segmento de habitantes mayores de 16 años y se delimita con las 
categorías:

a) Desarrollo humano.

b) Desarrollo económico.

c) Gestión comunitaria.

Las categorías a) y b), son la pauta para generar el diseño de cursos transdisciplinares, desarrollados en el contexto extra escolar, para la reanimación social simultáneamente con la solución de problemas y demandas específicas de los actores y colectivos comunitarios. De tal manera, que al diseño de cursos de capacitación tradicional, en materia agropecuaria, se incorporen temas transversales en desarrollo humano, con contenidos en empoderamiento, democracia, valores cívicos o resiliencia, según se demande. Ejemplificando este esbozo, enseguida se muestra el esquema de un curso modular, de autoría propia, que puede ser una guía para incluir la transversalidad referida.

\section{Esquema del curso modular con transversalidad.}

El diseño de este curso, de autoría propia, tiene el propósito de aportar tanto a la categoría de desarrollo humano como a la de desarrollo económico, sus contenidos responden a las condiciones actuales de un determinado contexto socio-productivo y que dada la complejidad de los fenómenos naturales, esas condiciones están sujetas a cambios imprevistos. Por tanto, el curso de capacitación y sus contenidos, pueden disminuir en su relevancia o perder su vigencia tan súbitamente como cambien las condiciones contextuales. En este entendido, a continuación se muestra el esquema del curso y posteriormente el diseño del mismo.

\begin{tabular}{|c|c|c|c|c|}
\hline Módulos & $\begin{array}{l}\text { Unidades de } \\
\text { aprendizaje }\end{array}$ & Contenidos & $\begin{array}{c}\text { Aprendizajes } \\
\text { del } \\
\text { capacitando }\end{array}$ & $\begin{array}{l}\text { Criterios de } \\
\text { evaluación }\end{array}$ \\
\hline \multirow{4}{*}{ 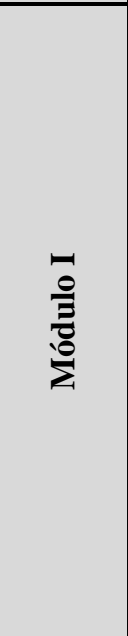 } & \multirow{2}{*}{$\begin{array}{l}\text { A. Título de la } \\
\text { unidad } \\
\text { de aprendizaje de } \\
\text { valores morales }\end{array}$} & $\begin{array}{l}\text { a) Contenido de la unidad } \\
\text { de aprendizaje } \mathrm{A} \text {. }\end{array}$ & $\begin{array}{l}\text { Aprendizaje del contenido a) } \\
\text { de la unidad de aprendizaje } \\
\text { "A". }\end{array}$ & $\begin{array}{l}\text { Menciona un mínimo } \\
\text { de } 3 \text { aspectos del } \\
\text { contenido a). }\end{array}$ \\
\hline & & $\begin{array}{l}\text { b) Contenido de la } \\
\text { unidad de } \\
\text { aprendizaje A. }\end{array}$ & $\begin{array}{l}\text { Aprendizaje del contenido b) } \\
\text { de la unidad de Aprendizaje } \\
\text { "A". }\end{array}$ & $\begin{array}{l}\text { Menciona } 2 \\
\text { Condiciones, como } \\
\text { mínimo, del contenido } \\
\text { b). }\end{array}$ \\
\hline & \multirow{2}{*}{$\begin{array}{l}\text { 1. Título de la } \\
\text { unidad de } \\
\text { aprendizaje del } \\
\text { aspecto } \\
\text { técnico del curso }\end{array}$} & $\begin{array}{l}\text { 1.1 Contenido de } \\
\text { la unidad de } \\
\text { aprendizaje } 1 .\end{array}$ & $\begin{array}{l}\text { Aprendizaje del contenido } 1.1 \\
\text { de la unidad de aprendizaje } 1 .\end{array}$ & $\begin{array}{l}\text { Menciona las } \\
\text { condiciones del } \\
\text { contenido } 1.1\end{array}$ \\
\hline & & $\begin{array}{l}1.2 \text { Contenido de la } \\
\text { unidad de aprendizaje } 1 .\end{array}$ & $\begin{array}{l}\text { Aprendizaje del contenido } 1.2 \\
\text { de la unidad de aprendizaje } 1 .\end{array}$ & $\begin{array}{l}\text { Menciona las } \\
\text { condiciones del } \\
\text { contenido } 1.2 \\
\end{array}$ \\
\hline
\end{tabular}




\begin{tabular}{|c|c|c|c|c|}
\hline \multirow{4}{*}{ 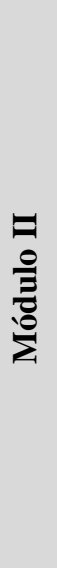 } & \multirow{3}{*}{$\begin{array}{l}\text { B. Título de la } \\
\text { unidad } \\
\text { de aprendizaje de } \\
\text { de } \\
\text { morales }\end{array}$} & $\begin{array}{l}\text { a) Contenido de la Unidad } \\
\text { de aprendizaje B. }\end{array}$ & $\begin{array}{l}\text { Aprendizajedel contenido } \\
\text { a) de la unidad de aprendizaje } \\
\text { B. }\end{array}$ & $\begin{array}{l}\text { Expone } \\
\text { los conceptos del } \\
\text { contenido a) }\end{array}$ \\
\hline & & $\begin{array}{l}\text { b) Contenido de la unidad } \\
\text { de aprendizaje B. }\end{array}$ & $\begin{array}{l}\text { Aprendizaje del contenido } \\
\text { b) de la unidad de aprendizaje } \\
\text { B. }\end{array}$ & $\begin{array}{l}\text { Menciona } \\
\text { aspectos del } \\
\text { contenido b). }\end{array}$ \\
\hline & & $\begin{array}{l}\text { c) Contenido de la unidad } \\
\text { de aprendizaje B. }\end{array}$ & $\begin{array}{l}\text { Aprendizaje del contenido } \\
\text { c) de la unidad de aprendizaje } \\
\text { B. }\end{array}$ & $\begin{array}{l}\text { Señala un mínimo } \\
\text { de } 3 \text { ejemplos } \\
\text { de aspectos } \\
\text { del contenido c). }\end{array}$ \\
\hline & $\begin{array}{l}\text { 2. Título de la } \\
\text { unidad }\end{array}$ & $\begin{array}{l}\text { 2.1 Contenido de la unidad } \\
\text { de aprendizaje } 2 \text {. }\end{array}$ & $\begin{array}{l}\text { Aprendizaje } \\
2.1 \text { de } \\
\text { aprendizaje } 2 .\end{array}$ & $\begin{array}{l}\text { Menciona } 3 \\
\text { condiciones del } \\
\text { Contenido } 2.1\end{array}$ \\
\hline
\end{tabular}

Esquema de curso modular, propuesto, con diseño propio.

\section{Diseño del curso modular}

Control y combate de la langosta migratoria (chapulín), en maíz de temporal. Con transversalidad en valores morales.

La plaga conocida como langosta migratoria, chapulín o chocho, causa severos daños en los cultivos de maíz sembrado en condiciones de temporal. Las mermas en las cosechas llegan a ser considerables, dependiendo del grado de infestación. El combate de la plaga, generalmente, se realiza cuando es muy notoria su presencia, sin embargo a los pocos días se observa nueva población de insectos jóvenes, lo cual hace suponer a los agricultores, que el insecticida aplicado favorece su reproducción. Ante esta situación, se hace necesario conocer las medidas para la prevención de la plaga, condiciones agroclimáticas para su reproducción, su ciclo de vida, taxonomía, insecticidas disponibles en el mercado, condiciones climáticas para aplicación de los insecticidas, medidas sanitarias para el manejo de insecticidas y tiempo post aplicación para cosechar.

\section{Temario específico}

\section{A) El trabajo en y con el grupo}

a. Actitudes que favorecen la convivencia. b. La armonía interior y su efecto grupal.

\section{Unidad 1. Reproducción de la langosta migratoria.}

1.1 Condiciones agroclimáticas para la reproducción.

1.2 Relación entre fecha de siembra y presencia de los insectos.

B) Valores, antivalores y actitudes.

a. Valores morales

b. Antivalores.

c. Actitudes positivas. 


\section{Unidad 2. Ciclo de vida y morfología}

2.1 Ciclo de vida

2.2 Morfología.

2.3 Comportamientos de interés.

C) La reconstrucción del tejido social

a. Tejido social.

b. Deterioro social.

c. Reconstrucción social

Unidad 3. Medidas de prevención de la plaga

3.1 Barbecho fitosanitario.

3.2 Limpia de cabeceras.

3.3 Limpia de acequias.

3.4 Uso de herbicidas.

\section{D) Reflejos del cambio actitudinal}

a. Apatía y solidaridad.

b. Dependencia y subsidiariedad.

c. Inactividad y actividad.

\section{Unidad 4. Control de la plaga}

4.1 Muestreo de población.

4.2 Insecticidas disponibles en el mercado local.

4.3 Características de los insecticidas disponibles.

4.4 Primera aplicación de insecticida.

4.5 Segunda aplicación.

4.6 Tiempo post aplicación, para la cosecha.

E) Impacto del cambio individual en el entorno

a. Desarrollo exógeno y desarrollo endógeno.

b. Incivismo y civismo.

\section{Unidad 5. Medidas sanitarias}

5.1 Almacenamiento y desecho de envases.

5.2 Manejo de utensilios y equipo.

5.3 Protección corpórea.

5.4 Ingesta de alimentos y bebidas.

5.5 Higiene personal. 


\section{Bibliografía sugerida}

\section{a). Bibliografía principal}

- CESAVEG. Programa de manejo integrado contra chapulín. Gobierno de Guanajuato. 2005.

- Rivera, C. Recomendaciones para el combate de plagas en maíz. En prensa.

- Savater, F. Ética para Amador. Editorial Ariel. España. 2008.

\section{b). Bibliografía complementaria}

- Beal, G. Bohlen, J. y Raudabaugh, Neil. Conducción y acción dinámica del

- grupo. Editorial KAPELUSZ. Buenos Aires. 1964.

- González, R. Los valores morales. Revista Cubana de salud pública. Vol. 21. Núm. 4. 2006. Pág. 269.

\section{Actividades de aprendizaje}

Conjeturas sobre las relaciones entre el ciclo biológico del chapulín y las condiciones agroclimáticas imperantes cuando aparecen las larvas, de igual manera: entre su ciclo de vida, morfología y las aplicaciones de insecticidas, así como entre las medidas de prevención y las condiciones agroclimáticas propicias para la eclosión.

\section{Reflexión sobre la práctica de valores morales y la integración grupal.}

\section{Actividades de apertura}

- Encuadre del curso.

- Socializar las interpretaciones de los participantes, sobre los términos del temario específico.

- Actividades de desarrollo

- Lectura comentada de la bibliografía básica. En cada sesión se alternan los contenidos: Se Inicia con los referentes a valores morales, para promover la integración del grupo. Continuar con los aspectos técnicos.

\section{Actividades de culminación.}

- Rememorar los temas y actividades de la sesión. Sinopsis de la próxima sesión. Salida.

- Evidencias de desempeño

\section{Unidad 1:}

Constatar la actitud asumida por el participante, en cada sesión. Registrando los aspectos favorables. 
Señalar la relación entre humedad y temperatura del terreno de labor, con la nacencia de los chapulines. Y su coincidencia con la época de siembra de maíz.

\section{Unidad 2:}

Describirán e identificarán in vivo los valores morales, antivalores, y actitudes positivas, argumentando su aplicación en la cotidianeidad.

Argumentar las fases propicias, en el ciclo de vida del chapulín, para la aplicación de insecticidas.

\section{Unidad 3:}

Explicar la composición del tejido social, los reflejos de su deterioro, y las alternativas para su reconstrucción.

Realizar las labores de prevención de la plaga. Exponiendo en qué casos se recurre a la aplicación de herbicidas.

\section{Unidad 4:}

Identificar y sustentar los cambios personales experimentados durante el transcurso de la capacitación.

Realizar el muestreo de campo y determinar el (los) insecticida(s) que se aplicará(n).

Referir el tiempo mínimo transcurrido desde la aplicación de insecticidas, para proceder a la cosecha, como prevención sanitaria.

\section{Unidad 5:}

Manifestar la contribución del individuo en la toma de decisiones, para contribuir en la mejora de la calidad de vida comunitaria, y mejorar su confianza en las instituciones y el respeto a los símbolos patrios.

Describir el procedimiento para manejo de envases, utensilios, equipo, ropa, mascarilla y sombrero, para evitar daños a las personas y al medio ambiente.

Plantear las alternativas para la ingesta de alimentos, en relación con el trabajo.

Externar lo conducente en casos de accidentes al manipular los insecticidas.

Argumentar la higiene personal, al finalizar las labores en el campo.

\section{Evaluación.}

Competente. Cuando el capacitando: Realiza las labores en correspondencia con los requerimientos de prevención y control de la plaga de la langosta migratoria. La operación de utensilios, equipo, ropa, mascarilla, sombrero, y envases, se realizan conforme a las 
especificaciones de su manejo. Se evidencia la ayuda mutua, la convivencia armónica y la disposición a la mejora de la calidad de vida.

No competente. Cuando se exhibe la deficiencia en alguno de los aspectos mencionados. Acciones remediales. Asistir al capacitando, para que desarrolle las competencias. Realizar nuevamente la capacitación.

\section{LISTA DE REFERENCIAS}

Acosta, M. Guía de Planeación y Control para la Formación de Productores en el Campo. Sep-Fondo de cultura económica. México. 1982.

Albert, M. La investigación educativa: claves teóricas. McGrawHill. México. 2007.

Gestiópolis. https://www.gestiopolis.com/que son crecimiento economico y desarrollo económico se relacionan/

Ander-Eg. Metodología y práctica del desarrollo de la comunidad. Lumen-Hvmanitas. Buenos Aires. 1998.

Bernal. Metodología de la investigación. Pearson Educación. Colombia. 2010.

Backer, T. La agricultura empresarial campesina. Instituto interamericano de cooperación para la agricultura. Lima. 1997. Recuperado de: https://books .google.com.mx/books?id=lucNAQAAIAAJ\&pg=PA21\&dq=Asistencia+tecnica +campesina\&hl=es-

419\&sa=X\&ved=0ahUKEwiczeL32ufbAhVPgK0KHWLYD2I

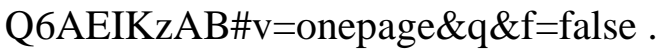

Bartle. Colectivo de potenciación comunitaria. 2011. http://cec.vcn.bc.ca/mpfc /indexs.htm .

Bureau Veritas. Manual para la formación en medio ambiente. Lex Veritas. Valladolid España. 2008.

Chevez, T. Las tres etapas del crecimiento espiritual. Palibrio Bloomingtoon

Comisión Mundial sobre el Medio y el Desarrollo. Declaración de Tokio. ONU Tokio. 1987. Recuperado de: file:///C:/Users/Usuario/Downloads/C OMISI\%C3\% 93N\%20MUNDIAL\%20SOBRE\%20EL\%20MEDIO\%20Y\%20EL\%20DESAR ROLLO.htm . 
Delval. El desarrollo humano. Siglo XXI. Madrid. 2008.

Gallegos. Educación holista. Pax. México. 1999.

Ferrer. Espiritualidad creativa. Kairós. Barcelona. 2003.

Freire. La educación como práctica de la libertad. Siglo XXI. México. 1969.

González y Diez. Educación en valores. Escuela española. Getafe, España. 1999.

Hernández. Metodología de la investigación. Mc Graw Hill. México. 2010.

Hurtado. El proyecto de investigación - Comprensión holística de la metodología y la investigación. Ediciones Quirón. Caracas. 2014.

ONU. Qué hacemos, Desarrollo. Recuperado de http://www.un.org/es/sections /what-wedo/promote-sustainable-development/ .

Papalia,Wendkos y Duskin. Desarrollo Humano. McGrawHill. México. 2010.Pdf.

Llorec, C. y otros. Como educar en valores. Narcea. Madrid. 1999.

Méndez. Problemas económicos de México. McGrawHill. México. 2008.

ONU. Informe de la conferencia de las Naciones Unidas sobre el Medio Humano. ONU. Nueva York. 1973. Recuperado de http://www.dipublico.org/ conferencias/mediohumano/ A-CONF.48-14-REV.1.pdf .

Wilber. El ojo del espíritu. Kairós. Barcelona. 2005. 